\title{
ALZHEIMER, DER UNAUSSPRECHLICHE DEUTSCHE ${ }^{1}$
}

\author{
Werner L. Heidermann ${ }^{1}$ \\ 1-Universidade Federal de Santa Catarina, Florianópolis, Santa Catarina, Brasil
}

\begin{abstract}
Zusammenfassung: Der Artikel führt verschiedene Romane zusammen, die von der Alzheimer-Erkrankung handeln. Im wesentlichen sind das David Wagners Der vergessliche Riese und Marcelo Rubens Paivas Ainda estou aqui, einer in deutscher Sprache, einer in brasilianischem Portugiesisch erschienener Roman. Es werden Indizien vorgestellt, die auf eine Art von Parallel-Logik im Universum von an Alzheimer Erkrankten hinweisen. Und es wird auf den vielfältigen Zusammenhang von Vergessen und Erinnern aufmerksam gemacht. Die Erinnerungen sind zum einen eher privater Natur (Wagner), zum anderen eher politischer und historischer Prägung (Paiva).
\end{abstract}

Palavras-chave: Mal de Alzheimer; David Wagner; Marcelo Rubens Paiva; Ditadura Militar

\section{ALZHEIMER, THE UNPRONOUNCEABLE GERMAN}

\begin{abstract}
The article brings together various novels dealing with Alzheimer's disease. Essentially, these are David Wagner's Der vergessliche Riese (The Forgetful Giant) and Marcelo Rubens Paiva's Ainda estou aqui (I am still here), one in German and one in Brazilian Portuguese. Evidence is presented that suggests a kind of parallel logic in the universe of people with Alzheimer's disease. And attention is drawn to the multifaceted connection between forgetting and remembering. The memories are on the one hand more of a private nature (Wagner), on the other hand they are more political and historical (Paiva).

Keywords: Alzheimer's disease; David Wagner; Marcelo Rubens Paiva; Military dictatorship
\end{abstract}

${ }^{1}$ So ist der Titel eines Kapitels in Marcelo Rubens Paivas Roman, auf das im Text eingegangen wird. 
Die Alzheimer-Krankheit ist das Thema von mittlerweile zahlreichen literarischen Werken. Stellvertretend werden hier David Wagners Der vergessliche Riese und Marcelo Rubens Paivas Ainda estou aqui untersucht. Es wird zu fragen sein, wie die Krankheit und sich aus ihr ergebende Lebensumstände erzählerisch gestaltet werden.

Krankheit ist eines der bedeutenden Universalthemen der Literatur. Krankheit ist hierbei nicht gleich Krankheit, und alle Krankheiten sind jeweils in ihrer Zeit verankert. Dietrich von Engelhardt, Medizinhistoriker: "Krankheiten werden keineswegs gleichmäßig oder in Übereinstimmung mit ihrer statistischen Verbreitung in literarischen Werken beschrieben. Psychische Leiden und Krebs nehmen eine hohe Stelle in der literarischen Nosologie der Gegenwart ein." (von Engelhardt 30)

Kein psychisches Leiden ist derzeit frequenter als die Demenz in ihren vielen Erscheinungsformen. Von 300.000 Diagnosen jährlich ist in Deutschland die Rede, ein großer Teil entfällt auf die Alzheimer-Erkrankung. ${ }^{2}$ In Brasilien geht man gegenwärtig von rund 1,2 Millionen an Alzheimer erkrankten Menschen aus, die mehrheitlich aber noch gar nicht diagnostiziert sind. ${ }^{3}$ Diese Häufigkeit schlägt sich in der Literatur nieder, in der englisch- und deutschsprachigen ${ }^{4}$, aber auch in der brasilianischen.

Rosvitha Friesen Blume hat 2015 zwei Romane miteinander in Beziehung gesetzt, die die Alzheimer-Krankheit in ihrem Zentrum haben: Arno Geigers Der alte König in seinem Exil und Heloisa

\footnotetext{
${ }^{2}$ Zahlen der Deutschen Alzheimer Gesellschaft(DAlzG), die 2018 von 1,7 Millionen an Demenz Erkrankten ausging. https://www.deutsche-alzheimer.de/ueber-uns/ presse/artikelansicht/artikel/neues-informationsblatt-der-deutschen-alzheimergesellschaft-alle-100-sekunden-erkrankt-in-deutsch.html\#: :text = Juni \% 20 2018., mehr\%20als \%20300.000\%20Neuerkrankungen\%20auf.

3 Angaben der Associação Brasileira de Alzheimer https://abraz.org.br/2020/ sobre-alzheimer/o-que-e-alzheimer-2/.

${ }^{4}$ Oft erwähnt werden Annette Pehnt: Haus der Schildkröten, Katharina Hacker: Die Erdbeeren von Antons Mutter, Bernd Eichmann: Vatter baut ab, Lisa Genova: Still Alice, J. Berlef: Hirngespinste. Aufzunehmen wären aber noch viele andere Werke auch aus der jüngsten Zeit, zum Beispiel Saša Stanišić: Herkunft.
} 
Seixas' O lugar escuro. Geiger wird mit den Worten zitiert, die die These von einem herausragenden Stellenwert dieser Krankheit bestätigen: "Von Alzheimer reden heißt, von der Krankheit des Jahrhunderts reden." (Geiger 58) Diese Erkenntnis ist relativ neu: In seinem wegweisenden Werk Lethe - Kunst und Kritik des Vergessens aus dem Jahr 2000 erwähnt Harald Weinrich Alzheimer gerade einmal an drei Stellen (und jeweils sehr kurz).

Wenn tatsächlich, wie oben behauptet wird, Krebs und psychische Erkrankungen in der Literatur frequenter sind als andere, so mag man eine Korrelation erahnen zwischen dieser Frequenz einerseits und dem Lebensbedrohlichen im Fall von Krebs bzw. dem Geheimnisvollen im Fall von Demenz andererseits. Dabei ist das Geheimnisvolle schwerer zu fassen als das Lebensbedrohliche.

Ein anderer wesentlicher Unterschied zwischen den beiden Typen von Krankheiten liegt in der literarischen Autorenschaft. Erfahrungen mit Krebs werden zumeist von den Betroffenen selbst dokumentiert; ${ }^{5}$ aus naheliegenden Gründen ist das bei DemenzErkrankungen anders. Hier ist es oft so, dass AutorInnen Alzheimer oder ähnliches bei einem nahen Angehörigen beschreiben, zumeist vom ersten Verdacht bis zu einer späteren Phase Jahre später. ${ }^{6}$ So ist es bei Arno Geiger, und so ist es bei zwei Autoren, deren Romane im Folgenden betrachtet werden sollen: David Wagners Der vergessliche Riese und Marcelo Rubens Paivas Ainda estou aqui. Wagner beschreibt die Erkrankung seines Vaters, Paiva die der Mutter. In Saša Stanišić' Herkunft beschreibt der Enkel die Demenz-Erkrankung der Großmutter.

Das Geheimnisvolle des Alzheimer-Leidens zeigt sich anfänglich nicht in Formen von Wahnsinn und Irrsinn, sondern in eher harmlos erscheinenden und leicht übersehbaren Auffälligkeiten im Verhalten der Personen. Familienangehörige von Alzheimer-Patienten wissen

\footnotetext{
${ }^{5}$ Stellvertretend für viele Titel sei hier Fritz Zorns Roman Mars aus dem Jahr 1977 genannt, der 1986 unter dem Titel Marte in Brasilien erschienen ist.

${ }^{6}$ Es gibt interessante Realisierungen: J. Bernlef, eigentlich Hendrik Jan Marsman beschreibt die Krankheit aus der Innensicht eines Alzheimer-Patienten. Der Roman heißt Hirngespinste.
}

Cad. Trad., Florianópolis, v. 41, no 3 p. 93-111, set-dez, 2021. 
Beispiele hiervon zu geben, Beispiele, die sich in einer Grauzone von noch belustigender und eher schrulliger Vergesslichkeit und längst besorgniserregendem Wirklichkeitsverlust abspielen und durchaus noch ihre amüsanten Momente haben können.

Arno Geigers Protagonist fragt beispielsweise bei der morgendlichen Anziehprozedur, nachdem sein Sohn ihm die Strümpfe gereicht hat, nach dem dritten Strumpf! Es scheint eine alternative Logik zu existieren; Geiger nennt sie "Privatlogik" (Geiger 52). 7 "Das Ja eines Dementen ist nicht immer auch ein Ja", heißt es bei Nicolai Glasenapp in einer Rezension des Buches Langsames Entschwinden von Inge Jens. Marcelo Rubens Paivas Verständnis geht in eine ähnliche Richtung, wenn er den Gerichtstermin beschreibt, in dessen Verlauf seine Mutter den Sohn zu ihrem Vormund bestimmt und zu dem Schluss gelangt: "Und vielleicht haben wir ja nicht nur EIN Gedächtnis." (Paiva 23) ${ }^{8}$

Im Folgenden soll in den beiden genannten Werken genau diese "Privatlogik" untersucht werden sowie die Frage, wie mit ihr erzählerisch umgegangen wird. Dieser Umgang mit der Krankheit und den Kranken scheint nämlich den Grad des Leidens und Mitleidens seitens der Angehörigen mitzubestimmen. Eine These könnte sein, dass verloren ist, wer gegen die alternative Logik des Kranken anargumentiert, und dass nur einigermaßen gesund bleibt, wer die konventionelle Logik partiell aufgibt und sich auf ein ganz anderes Universum von Erinnerungen und Nicht-Erinnerungen, von bildlichen Verbindungen und gedanklichen Verflechtungen einstellt. Paivas Ainda estou aqui und Wagners Der vergessliche Riese können hier Hinweise geben.

7 Vielleicht gibt es sogar eine Kryptologik in den Herstellungsvorgängen von Büchern. Arno Geigers Vater heißt August Geiger. Im Klappentext der brasilianischen Ausgabe wurde aus August durchgehend Auguste. Auguste Deter (1850-1906) war die Patientin Alois Alzheimers, deren Krankheitsbild dann nach dem Arzt benannt wurde.

8 "E talvez não tenhamos UMA só memória." (23) 


\section{David Wagners Der vergessliche Riese}

Ein Beispiel für die hin und wieder durchscheinende Absurdität in der Logik von Protagonisten der Demenz-Literatur ist das folgende: Rudolf Dublany, ehemals freiberuflicher Dozent für "Interkulturelles Management und Verhandlungstraining für Autokonzerne und für den Deutschen Entwicklungsdienst" (Wagner 246), greift nach dem Unterarm seines Sohnes David, "zieht ihn zu sich und sagt: 'Das ist doch meine Uhr, die du da trägst!' - 'Papa, das sagst du jedes Mal, wenn ich dich besuche. Du hast sie mir geschenkt, vor über zehn Jahren.' - 'Wirklich? Und ich dachte schon, es wäre mein Arm, der da auf dem Tisch liegt. "' (Wagner 218)

Die Beschreibungen sind voll von dieser Art von Provokation des gesunden Menschenverstandes. David Wagners Der vergessliche Riese stellt aber in gewissem Sinn auch eine Ausnahme dar. Wir lesen einen Roman, der nicht nur die krassesten Endstadien der Erkrankung ausspart; die erzählerische Konstruktion insgesamt fundiert mehr auf einer Heiterkeit als Grundstimmung als auf der Darstellung von irritierendem Persönlichkeitsverlust. Diese Grundstimmung ergibt sich durch die beinahe durchgehende dialogische Struktur: Vater, nur etwas über 70, und Sohn, um die 40, verbringen Zeit miteinander, und zwar anlässlich von insgesamt neun Treffen, im Laufe von rund vier Jahren, geschildert in neun Kapiteln. Die Begegnungen sind nicht sonderlich konfliktreich; Vater und Sohn haben eine gute Zeit miteinander. Der Sohn spricht den Vater mit "Papa" an; der Vater den Sohn durchgehend mit "Freund".

Die Heiterkeit im Umgang der beiden mag befremden. Dublany wird zwar zunehmend vergesslicher, was ihm auch bewusst ist. Er wiederholt unablässig offensichtlich ihn prägende Äußerungen ("Die Dublany sind sehr intelligent, im Alter aber werden sie alle blöd.") (Wagner 10) Er vergisst Daten, verlegt Gegenstände, verwechselt Situationen, erinnert sich nicht an Namen, an seine eigene berufliche Tätigkeit, an die eigene Adresse; er weiß sich 
aber ausnahmslos korrekt und verständlich $\mathrm{zu}$ artikulieren und redet nicht wirklich Unsinn. Gegen Ende reflektiert er seine Situation und fasst sie in Form eines Bildes: “'Oft komme ich mir vor, als wäre ich aus einem Buch gefallen und könnte nicht zurück. Ich bin plötzlich in einer ganz anderen Geschichte und weiß nicht, was ich da soll. "' (268) Die Konflikte in Wagners Roman spielen sich sozusagen auf dem hohen Niveau der Bonner Senioren-Residenz ab und haben nicht die destruktive Strahlkraft anderer Demenz-Leidensgeschichten. Ein Beispiel: Vater: “'Einen braunen Hut zum schwarzen Mantel? Das passt doch nicht. "' Sohn: "Ausnahmsweise, bitte. " (Wagner 248)

Der Sohn treibt sogar seinen Schabernack mit der Vergesslichkeit des Vaters. "'Deine Mutter war meine erste Frau, oder ? Sie hieß ... sie hieß ... - ja wie hieß sie noch mal? Und wie hieß meine zweite Frau?' - "Sie hieß ... - habe ich vergessen, Papa.'” (Wagner 172) Es gibt eine amüsante Komplizenschaft, die im Scherz das Leiden der Vergesslichkeit erträglich sein lässt. Und es gilt, was Arno Geiger in seinem Roman vom alten König schreibt: "Das ist sozusagen das Gegenteil dessen, was man normalerweise über die Alzheimer-Krankheit sagt - dass sie Beziehungen zerstört. Manchmal stellt sie Beziehungen her.” (Geiger 185)

So wenig diese Krankheit Verharmlosung und Beschönigung erlaubt, so sicher ist, dass sie, zumindest in den ersten Phasen, durchaus auch Leichtigkeit und einen gewissen Unernst zulässt. Dublany ist zweifelsfrei an Alzheimer erkrankt; das haben neurologische und psychiatrische Untersuchungen belegt. Er ist aber imstande, sich zu reflektieren und seine Situation zu verbalisieren: “'Du kannst mir viel erzählen. Ich weiß nichts mehr. Und ich sage dir, so schlecht ist das gar nicht." (Wagner 151) Hin und wieder verblüfft er durch Verhaltensweisen, die man nicht gerade mit dem Alzheimer-Leiden assoziiert. In einem China-Restaurant redet er ein paar Sätze Mandarin mit dem Kellner, was den Sohn anerkennend sagen lässt: “'Du bist voller Überraschungen.'” (Wagner 262) 
Dem Vergessen etwas Gutes abgewinnen, das versucht auch die demente Großmutter in Saša Stanišić Herkunft, ausgezeichnet mit dem Deutschen Buchpreis 2019, wenn sie auf die Frage des Neffen “'Wo bist du zu Hause?'” antwortet: “'Begreif das endlich. Es zählt nicht, wo was ist. Oder woher man ist. Es zählt, wohin du gehst. Und am Ende zählt nicht mal das. Schau mich an: Ich weiß weder, woher ich komme, noch wohin ich gehe. Und ich kann dir sagen: Manchmal ist das gar nicht so schlecht.'" (Stanišić 337)

\section{Marcelo Rubens Paivas Ainda estou aqui}

Konflikte zwischen an Alzheimer Erkrankten und ihren PflegerInnen und Angehörigen sind oft Konflikte zwischen konventioneller und nicht konventioneller Logik. Es findet sich ein Beispiel bei Paiva, wie diese Konflikte offensichtlich am ehesten aufgelöst werden können. "Einverstanden sein, das war das Beruhigungsmittel, das uns gefehlt hatte. Einverständnis wurde zu unserem Heilmittel. Einverständnis machte Schluss mit den Diskussionen. Sie beruhigte sich. Lachte sogar. Und dachte an andere Dinge. Dieser Prozess des Zusammenlebens mit einem unruhigen Geist und seinen Höhen und Tiefen und ohne Logik, speziell bei einem Menschen, dessen Leben in den Bahnen der Logik verlaufen ist, und auch, wie man dem Trotz mit Witz begegnet, das alles war ein Geheimnis, das wir beinahe zufällig mit der Zeit gelernt haben." 9

Paiva sagt dasselbe an anderer Stelle in ganz anderen Worten, und zwar in einem Bild, das für einen Autor aus Rio de Janeiro mehr als einleuchtend ist: "Wer schon mal auf dem offenen Meer

9 "Concordar passou a ser o tranquilizante que faltava. Concordar era nosso remédio. Concordar acabava com a discussão. Ela se acalmava. Até ria. E pensava em outra coisa. Esse processo de convívio com o gênio instável, de altos e baixos e sem lógica, especialmente de alguém que seguiu a vida nos trilhos da lógica, e de como transformar a inconformidade em piada, foi um segredo que aprendemos quase casualmente com o tempo." (238) 
geschwommen ist, der weiß: statt verzweifelt gegen die Strömung anzukämpfen, ist es besser, sich für ein paar Augenblicke treiben zu lassen; man braucht Ruhe und Mut; die Strömung nimmt an Stärke ab, und man entkommt ihr." 10

Holger Helbig behauptet, “'daß Alzheimer eine besonders literarische Krankheit' sei und besondere Geschichten hervorbringe”, bevor er ausführt: „Alzheimer zwingt die Literatur zur Selbstreflexion. Zu den Besonderheiten der Krankheit gehört nicht nur, daß sie mit Notwendigkeit hochgradig literarisierte Krankheitsbeschreibungen verursacht, sondern vor allem, daß sie die anthropologische und kulturelle Funktion des Erzählens auf eine Weise betont, die Literatur und Erzähltheorie nicht werden ignorieren können. “ (Konrad-Adenauer-Stiftung)

Es muss an dieser Stelle darauf hingewiesen werden, dass es bei der Analyse und Reflexion von Literatur zum Demenz/AlzheimerKomplex nicht darum gehen kann, ein Leiden zu intellektualisieren! Demenz und Alzheimer sind grausame Krankheiten, die relativ einfach beschreibbar, relativ sicher diagnostizierbar und abbildbar und dabei nicht heilbar sind. In den Romanen von Wagner und Paiva stehen Kranke im Mittelpunkt, die sich im Anfangsstadium und in der Folge in einem fortgeschrittenen Stadium der Krankheit befinden. Paiva und Wagner verzichten auf die literarische Bearbeitung der letzten der vier von Paiva skizzierten Phasen (initial, moderat, schwer, terminal) ${ }^{11}$.

Beide Autoren verzichten also auf die Beschreibung des völligen Kontroll- und Persönlichkeitsverlusts, genau wie Arno Geiger - und anders als etwa Tilman Jens in Demenz. Abschied von meinem Vater, der heftig für die schonungslose Darstellung der zunehmenden und schließlich vollständigen Orientierungslosigkeit seines Vaters kritisiert worden ist. Wagner und Paiva verweigern sich auch einer

10 "Quem nadou em mar aberto sabe: antes de lutar desesperadamente contra a correnteza, é melhor deixar-se levar por instantes; é preciso ter calma e coragem; a correnteza, então saímos fora." S. 35

11 "forma inicial, moderada, grave, terminal" Paiva S. 232 
jedweden Behauptung von Kausalität der Erkrankung, ebenfalls im Unterschied zu Jens, der eine Beziehung zwischen dem Leiden des Vaters und einer kurz vor der Erkrankung gemachten Entdeckung sieht. Der Sohn glaubt an einen Zusammenhang zwischen dem Vergessen und dem vorherigen Verheimlichen der (möglichen, wohl letztlich nicht bewiesenen) NSDAP-Mitgliedschaft von Walter Jens.

\title{
Erinnern und Vergessen
}

Ulrike Vedder stellt diese Interpretation in einen größeren Zusammenhang:

\begin{abstract}
Während große Teile der deutschsprachigen Literatur der 1990er Jahre im Zeichen des historischen, kulturellen und familialen Gedächtnisses sowie der Verhältnisbestimmung zwischen Erinnern und Erzählen standen, lässt sich für die jüngere Zeit ein vermehrtes Interesse am Vergessen konstatieren - ein Vergessen, das im Format der Familienund Generationenromane, aber auch in eher avantgardistisch verfahrender Literatur thematisiert und inszeniert wird und das vielfach an Demenzerkrankungen der Protagonisten gebunden ist. (Vedder 274)
\end{abstract}

Weniger als die Krankheit steht im Zentrum von Wagners Roman eine Vater-Sohn-Beziehung, deren von beiden Seiten gepflegte Lockerheit und Souveränität anrühren. Der Vater vergisst nach und nach mehr und mehr. Ganz am Ende merkt er, dass er nicht am Nil wohnt, sondern in Bonn am Rhein, um gleich nachzufragen: "'Wie heißt der Fluss noch mal? Und jetzt weiß ich wieder, was ich dich fragen wollte, Freund: Wer sind eigentlich deine Eltern? ," (Wagner 269)

Es gibt keine Versuche, Kausalitäten zu konstruieren; wohl aber scheint auch bei Wagner die deutsche Geschichte durch. Der Vater 
tut sich schwer mit dem Aufzählen der Namen seiner insgesamt acht Geschwister. Der Sohn hilft ihm: “'Deine Geschwister heißen oder hießen Hans, Hase, Hermann, Norbert, Gunter, Wellgunde, Floßhilde und Woglinde. Deine Kinder aber heißen Hanna, Miriam und David. Fällt Dir da was auf? '" Und der an Demenz erkrankte Vater gibt folgende Antwort:

\begin{abstract}
'Wir mussten euch Kindern jüdische Vornamen geben, weil unsere Eltern alle Juden umgebracht hatten. Weil keine Juden mehr da waren. [...] Deine Mutter und ich, wir dachten, das wäre eine Geste. Eine wichtige Geste. Heute weiß ich, dass dahinter auch unser Wunsch steckte, Opfer, nicht Täter zu sein. Und dass es nur die andere Seite der deutschen Gründlichkeit war: erst alle Juden umbringen und dann, weil sie uns fehlen, den eigenen Kindern jüdische Namen geben. Als ließe sich so etwas wiedergutmachen. Er seufzt. (Wagner 196f.)
\end{abstract}

Wir lesen eine glasklare Begründung mit einer zynischen Kommentierung; in dieser Phase der Erkrankung ist der Patient Dublany, ehemals Opernfreund und Wagner-Kenner, in seinen lichten Momenten zu Scharfsinn und Ironie fähig, Dispositionen, die man üblicherweise eher nicht mit der Alzheimer-Erkrankung verbindet.

Marcelo Rubens Paiva beschränkt sich ebenfalls nicht auf die literarische Darstellung der Alzheimer-Demenz. Die Erkrankung seiner Mutter Eunice (1932-2018) bildet vielmehr den Rahmen für ein anderes Thema und die Rolle, die seine Mutter dort gespielt hat: Es geht wesentlich um die Militärdiktatur im Brasilien der Jahre 1964 bis 1985 und um die Umstände der Verschleppung, Folterung und Tötung von Rubens Beyrodt Paiva (1929-1971), Eunices Mann, Vater des Autors und ehemaliger Abgeordneter des brasilianischen Parlaments. Die ersten und letzten Kapitel behandeln auf rund 80 Seiten die Erkrankung der Mutter, während Paiva auf weit über 200 Seiten dem Verschwinden seines Vaters nachspürt sowie der ganz kurzen Inhaftierung der 
Mutter, die wiederum als ihre Lebensaufgabe ansieht, ihrem Mann Gerechtigkeit widerfahren zu lassen und verantwortliche Militärs zur Rechenschaft zu ziehen. Marcelo Rubens Paiva schreibt 200 Seiten moderner brasilianischer Geschichte aus der Perspektive desjenigen, der als Zwölfjähriger seinen Vater an die Militärdiktatur verloren hat und seine Mutter in ihrem Kampf um Aufklärung unterstützt und sie sein Leben lang für ihren Kampfgeist, ihre Unabhängigkeit, ihre Willensstärke und nicht zuletzt für ihre liberalen Ansichten bewundert.

"Ihr Stolz war größer als ihre Vergesslichkeit." 12 - so die Schlussfolgerung des Sohnes, der die Erkrankung der Mutter ab ihrem 77. und bis zum 85. Lebensjahr dokumentiert. "Ich bin noch hier. Noch bin ich hier. - Ja, du bist hier, noch bist du hier. Meine Mutter, 85 Jahre alt, trat nichts ins vierte Stadium, dem schlimmsten von allen. Ihr Leben hat viele Akte. Wir werden einen Akt mehr haben. Während der Tod meines Vaters kein Ende hat."13

Alzheimer tritt als "Der unaussprechliche Deutsche"14 (Paiva 225) auf; Eunices Nachbar, ebenfalls an Alzheimer erkrankt: “'Der verfluchte Deutsche [...]' Wenn er etwas vergaß, sagte er: 'Lass mich in Ruhe, du Deutscher, hau ab! ""15 (Paiva 240) Die Mutter erwähnt den Namen Alzheimer kein einziges Mal (Paiva 261).

Mehr als wohl in vielen anderen Werken zum Thema tritt bei Paiva die Lebensleistung der Mutter in den Vordergrund und überstrahlt die Desorientiertheit der letzten Jahre. Weniger als eine immer bedrückende Darlegung des Ich-Verlusts ist es die liebevolle Würdigung der Mutter, die viele Möglichkeiten hatte, sich um ihren Sohn zu sorgen. Marcelo rekapituliert in einem anderen Roman,

\footnotetext{
12 "Seu orgulho era maior do que seu esquecimento." S. 262

13 "Eu ainda estou aqui. Ainda estou aqui. Sim, você está aqui, ainda está aqui. Minha mãe, aos oitenta e cinco anos, não entrou no Estágio IV, o pior de todos. Sua vida tem muitos atos. Teremos mais um. Enquanto a morte do meu pai não tem fim. S. 262f. - Eunice Paiva stirbt 2018.

${ }^{14} \mathrm{O}$ alemão impronunciável (S. 225)

${ }^{15} \mathrm{O}$ maldito almão [...] Quando ele se esquecia algo, falava: - Me deixa em paz, alemão, sai! (240)
} 
Feliz ano velho (Schönes altes Jahr), den Badeunfall, der zu seiner Querschnittslähmung führte, der die Mutter in sehr resoluter Weise begegnete:

Sie ist meine Mutter, die meine Bronchitis-Anfälle behandelt hat, sich um meinen jugendlichen Leichtsinn gekümmert, mich auf der Intensivstation versorgt hat, in Krankenhäusern, die über anstehende Operationen verhandelt hat, die sich nicht in meine Pläne oder literarischen Projekte eingemischt, die meine Hand massiert hat, um eine Atrophie zu verhindern, die meine ersten Texte Korrektur gelesen hat, auch meine ersten Bücher, die über Jahre meine Steuererklärung erledigt und die meine Verträge geprüft hat, die mich zur $\mathrm{AACD}^{16}$ gebracht hat, mich in Rio beherbergt, die nicht eine meiner Film- und Theater-Premieren und Buchvorstellungen ausgelassen hat, die an meiner Seite Interviews gegeben hat, die mich erzogen hat und die Brasilien dahin gebracht hat, sich infrage stellen lassen. ${ }^{17}$

In
Alzheimer-Erzählungen im familialen Generationenroman [...] geht es um Abhängigkeitsverhältnisse aufgrund des Altersunterschieds und der familialen Konstellation, d. h. um Asymmetrien der Beziehung, aber auch um

\footnotetext{
${ }^{16}$ Mit $A A C D$ ist die Associação de Assistência à Criança Deficiente gemeint, eine Organisation, die sich in verschiedenen Städten Brasiliens der Betreuung behinderter Kind annimmt. https://aacd.org.br/

17 "Ela é minha mãe, ela cuidou dos meus ataques de bronquite, da minha inconsequência juvenil, tratou de mim na UTI, em hospitais, negociou operações, não interferiu nos meus planos ou projetos literários, massageou a minha mão para não atrofiar, fez a revisão dos meus primeiros textos, inclusive dos meus primeiros livros, fez meu imposto de renda por anos, reviu meus contratos, me levou para a AACD, me hosüpedou no Rio, não perdeu uma estreia de filme, peça ou lançamento de livro meu, deu entrevistas ao meu lado, me ensinou e fez o brasil repensar." (Paiva, S. 260)
} 
Rollentausch, wenn die Kinder die Verantwortung für ihre hilflosen Eltern übernehmen müssen, an die sie durch deren Orientierungslosigkeit und Schwäche gebunden sind, so dass die Generationsfunktionen sich verschieben. (Vedder 279)

Dieser Rollentausch wird als schmerzhaft und gemeinhin als Überforderung empfunden. Von einer "Unabgegoltenheit der welchselseitigen Wünsche zwischen den Eltern und ihren alternden Kindern" (Vedder 280), die Vedder in Alzheimer-Erzählungen diagnostiziert, kann weder bei Wagner noch bei Paiva, und wohl auch nicht bei Arno Geiger, die Rede sein. David Wagner übrigens hat sich, wie Paiva mit der Querschnittslähmung, in einem anderen Roman mit einem anderen Trauma auseinandergesetzt: seiner lebensbedrohenden Autoimmunhepatitis mitsamt einer Lebertransplantation. Beide waren also in gewisser Weise gestählt, als sie sich an den Komplex Gesundheit-KrankheitSterben-Tod machten.

AndieStelle von Unabgegoltenheitsskrupelnund Schuldgefühlen, Überforderung und Hilflosigkeit treten bei Paiva Respekt vor der Lebensleistung der Mutter im Privaten wie im Politischen und bei Wagner eine heitere Gelassenheit in der Sicherheit, dass der Vater gut und professionell versorgt wird.

Wagner verzichtet ganz, Paiva weitgehend auf medizinische Stellungnahmen und Diagnosen. In beiden Fällen ist zwar von den wohl üblichen Tests die Rede ("Welcher Tag ist heute?" - "Wie heißt der Staatspräsident?"), wesentlich sind aber in beiden Fällen nicht psychiatrische Sichtweisen, sondern viel eher die der Familienangehörigen. Paiva zum Beispiel zitiert keine Fachleute zu der Frage nach dem bei Alzheimer-Kranken zu beobachtenden mehrfachen Wiederholen. Er stellt stattdessen eigene Überlegungen an:

Sich wiederholen ist eine der Routinen von Menschen mit

Demenz. Ich weiß nicht, ob das daher kommt, dass die 
Person das, was sie gesagt hat, sofort wieder vergessen hat, oder ob sie das, was sie sagen will, unterstreichen will, weil einige ihr keine Aufmerksamkeit schenken. (Paiva 28f.)

Dieses Verständnis entspricht der subjektiven Herangehensweise des Autors, dem es weniger um eine Aufklärung in Sachen Demenz geht, sondern um die Würdigung seiner Mutter in ihrem persönlichen, aber auch politischen und historischen Kontext, und zwar zu einem Zeitpunkt, den sie noch erlebt - daher der Titel (Ainda estou aqui/Noch bin ich hier).

Die an Alzheimer erkrankte Mutter ist dem Sohn schon früh "die eigentliche Heldin der Familie" 18 (Paiva 38). Schon zu Beginn ihres politischen Kampfes gegen die Diktatur, unmittelbar nach der Entführung des Mannes, schärft sie ihren Kindern ein:

Unser Feind würde uns nicht besiegen. Die Familie Rubens Paiva weint nicht vor den Kameras, macht nicht auf Mitleid, stellt sich nicht als Opfer dar und nicht als Revanchist. [...] Die Familie Rubens Paiva ist nicht das Opfer der Diktatur, das Land ist Opfer der Diktatur. Das Verbrechen war gegen die Menschlichkeit, nicht gegen Rubens Paiva. Wir müssen gesund sein und braun gebrannt für die Gegenoffensive. Angst, Tränen, Hass, all das nur in den eigenen vier Wänden. Meine Mutter gab den Ton an, sie hat uns erzogen. ${ }^{19}$

Paiva zeigt nicht in der Hauptsache Vergessenund Vergesslichkeit seiner Mutter, sondern seine Mutter als Frau, die in der Zeit vor

\footnotetext{
18 "a verdadeira heroína da família"

${ }^{19}$ Nosso inimigo não iria derrubar. Família Rubens Paiva não chora na frente das câmeras, não faz cara de coitado, não se faz de vítima e não é revanchista. [...] A família Rubens Paiva não é a vítima da ditadura, o país que é. O crime foi contra a humanidade, não contra Rubens Paiva. Precisamos estar saudáveis, bronzeados para a contraofensiva. Angústia, lágrimas, ódio, apenas entre quatro paredes. Foi a minha mãe quem ditou o tom, ela quem nos ensinou." S. 39
} 
ihrer Erkrankung dem Militärregime die Stirn geboten hat. Sie ist gerade aus kurzer Haft entlassen; ein Fotojournalist braucht eine Aufnahme der Familie, Mutter und fünf Kinder: "Ernster! Trauriger! Unglücklicher! Wir schafften es nicht. Oder wollten es nicht. Der Protest hat uns immer inspiriert. Heute betrachte ich das Foto und sehe in den Augen meiner Mutter: Wer glaubst du, dass du bist, um uns unglücklich zu machen?" 20

Es muss hier kurz darauf hingewiesen werden, dass der Vater des Autors eines der prominentesten Opfer der Militärdiktatur war, dass sich die mittleren Kapitel des Romans streckenweise wie das Who is who? der brasilianischen Linken der Zeit liest und dass der Roman selbst einen Einblick in das Selbstverständnis und Selbstbewusstsein der Linken während der Diktatur gibt. Es geht bis hin zu Lebensgewohnheiten wie beispielsweise dieser Auszug skizziert: "man verachtete das Fernsehen und schätzte Bücher, kein Luxus, Möbel aus Leder und Holz, keine Bilderrahmen, eine Sicherheitsmaßnahme, die die brasilianische Linke klugerweise und wie selbstverständlich während der Diktatur ergriff" ${ }^{21}$.

Das Ende der Diktatur wird mit dem Jahr 1985 datiert; Paiva zitiert mehrfach den Ato Constitucional AI-5, das Dekret, das 1968 erlassen wurde und die rigideste Phase der Militärdiktatur einleitete. Unter anderem führte das Dekret zur Schließung des Parlamentes. Genau diese Maßnahme macht die Diskussion des AI-5 so aktuell, wird doch in den letzten Jahren mehrfach mit einem "AI-5" geliebäugelt, und zwar von höchster Stelle, zum einen vom Staatspräsidenten Jair Bolsonaro, zum anderen von seinem Sohn Eduardo. Der nicht widersprochene Ruf nach einem "neuen AI-5" ist die gefährliche Konsolidierung der extremen Rechten.

\footnotetext{
20 "fiquem mais sérios, mais tristes, mais infelizes. Não conseguimos. Ou não queríamos. A irreverência sempre nos inspirou. Observo a foto hoje e vejo nos olhos da minha mãe: quem você pensa que é, para nos fazer infelizes?" S. 252 ${ }^{21}$ desprezo por TV, prioridade aos livros, nada de luxo, móveis de couro e madeira, nada de porta-retratos, uma medida de segurança que a esquerda brasileira adotou sábia e espontaneamente durante a ditadura" S. 139
} 
Paiva mahnt nicht, aktualisiert nicht! Er stellt das Drama seiner Familie dar, das im Verschwinden seines Vaters gipfelte und den bis heute nicht wirklich aufgeklärten Umständen seiner Tötung. Seine Leiche ist nie aufgefunden worden. Ainda estou aqui ist Dokumentation des Vergessens seitens der Mutter und gleichzeitig das Versprechen, die Erinnerung sowohl an den Vater als auch an die Gräuel des Unrechtsregimes wachzuhalten. Paiva dokumentiert sehr unangestrengt und ohne Pathos, lässt dabei große Mengen an Details einfließen, beispielsweise das Schicksal von Maria Auxiliadora Lara Barcelos, einer Kämpferin im bewaffneten Widerstand, Opfer von Verfolgung und Folter, die sich 1976 in Berlin das Leben nahm. Vergessen hier, Erinnerung dort.

Paiva führt aus, dass und warum eine Frage wie "Erinnerst du dich an mich?" Alzheimer-Kranke in Panik versetzen kann. Seine Mutter antwortet jeweils nur sehr lakonisch und uneindeutig, und einmal spricht ihre Tochter, Rubens' Schwester, stellvertretend für die Mutter, indem sie sich einer Art alternativer Logik bedient und die Frage eines Lehrers "Erinnerst du dich an mich?" so beantwortet: "Das ist nicht wichtig, fundamental ist nicht die Erinnerung, sondern die Frage, wie du heute mit ihr umgehst." 22

\section{Schluss}

Der Artikel hat versucht, anhand von zwei Romanen, einem in deutscher Sprache, dem anderen in portugiesischer Sprache, nämlich David Wagners Der vergessliche Riese und Marcelo Rubens Paivas Ainda estou aqui nachzuvollziehen, inwiefern nicht so sehr Vergessen und Leiden und Krankheit im Zentrum der erzählerischen Gestaltung stehen, sondern das, was der Erzähler für erinnernswert hält. In Wagners Fall mag es am ehesten die Liebenswürdigkeit sein, die den gesunden Vater auszeichnete

22 “Não é importante, o fundamental não é a lembrança, mas como você interage com ela hoje.” S. 192

Cad. Trad., Florianópolis, v. 41, no 3 p. 93-111, set-dez, 2021. 
und die dieser auch als Kranker zu bewahren weiß; in Marcelo Rubens Paivas Roman ist es die starke Persönlichkeit der Mutter vor dem Hintergrund grauenhafter Ereignisse während der brasilianischen Militärdiktatur. In beiden Werken wird auf drastische Beschreibungen von Krankheitsverläufen verzichtet. In beiden Romanen materialisiert sich größter Respekt gegenüber der Mutter, bzw. dem Vater. Die Arbeit hat Indizien für eine gewisse Parallel-Logik bei den beiden Alzheimer-Kranken aufgezeigt, die dem sie interpretierenden Umfeld Geduld und Phantasie abverlangt.

\section{Literatur}

Berlef, J. (eigentlich Hendrik Jan Marsman): Hirngespinste. Übersetzt (aus dem Niederländischen) von Maria Csollány. Ditzingen: Reclam, 2016.

Blume, Rosvitha Friesen: "Loucura ou exílio? A literarização da demência materna e paterna por Heloisa Seixas e Arno Geiger”. In: Atas do I Congresso da Associação Brasileira de Estudos Germanísticos. São Paulo 2015, pp. 77-84. http://germanistik-brasil.org.br/?page_id $=861$

Eichmann, Bernd: Vatter baut ab. Random House eBook 2014.

Geiger, Arno: Der alte König in seinem Exil. München: Carl Hanser Verlag 2011.

Genova, Lisa: Still Alice. Mein Leben ohne Gestern. Übersetzt (aus dem Englischen) von Veronika Dünninger. Bergisch Gladbach: Bastei Lübbe 2015.

Glasenapp, Nicolai: “Über den Umgang mit Dementen” https://literaturkritik.de/ $\mathrm{id} / 22316$ - Zugriff am 10.1.2021 
Hacker, Katharina: Die Erdbeeren von Antons Mutter. Frankfurt am Main: Fischer 2010.

Jens, Tilman: Demenz. Abschied von meinem Vater. Gütersloh: Gütersloher Verlagshaus 2009.

Konrad-Adenauer-Stiftung: Medizin und Literatur. Eichholzer Fachtagung der Konrad-Adenauer-Stiftung 14.-16. November 2004. www.kas. de`doc`kas_5739-544-1-30-Zugriff am 10.2.2021

Paiva, Marcelo Rubens: Feliz ano velho. Rio de Janeiro: Alfaguara 2015 (1982).

Paiva, Marcelo Rubens: Ainda estou aqui. Rio de Janeiro: Alfaguara 2015.

Pehnt, Annette: Haus der Schildkröten. München: Piper 2008.

Saša Stanišić: Herkunft. München: Luchterhand Literaturverlag 2019.

Vedder, Ulrike: "Erzählen vom Zerfall. Demenz und Alzheimer in der Gegenwartsliteratur". In: Zeitschrift für Germanistik. Neue Folge, vol. 22, $\mathrm{n}^{\mathrm{0}}$. 2 (2012) Frankfurt am Main: Peter Lang. jstor.org/stable/23979247?seq=1 Zugriff am 1.2.2021.

Von Engelhardt, Dietrich: "Der Kranke und seine Krankheit in der Literatur". In: Doerr, Wilhelm/Schaefer, Hans/Schipperges, Heinrich: Der Mensch in seiner Eigenwelt. Anthropologische Grundfragen einer Theoretischen Pathologie. Springer-Verlag. https://link.springer.com/chapter $/ 10.1007 \%$ 2F978-3-642-84612-0_3 - Zugriff am 4.12.2021.

Wagner, David: Der vergessliche Riese. Hamburg: Rowohlt Verlag 2019.

Wagner, David: Leben. Reinbek: Rowohlt 2013.

Weinrich, Harald: Lethe - Kunst und Kritik des Vergessens. München: Beck 2000. 
Zorn, Fritz: Mars. München: Kindler Verlag 1977.

Zorn, Fritz: Marte. Übersetzung von Walter Wehrs. Rio de Janeiro: Nova Fronteira, 1986.

Recebido em: 01/05/2021

Aceito em: 30/07/2021

Publicado em setembro de 2021

Werner L. Heidermann. Email: heidermann@gmail.com. ORCID: https://orcid. org/0000-0003-3968-6460. 